\title{
Proton decay and nuclear dynamics
}

\author{
M. Alvioli, ${ }^{1}$ O. Benhar, ${ }^{2}$ M. Ericson, ${ }^{3}$ and M. Strikman ${ }^{1}$ \\ ${ }^{1} 104$ Davey Lab, The Pennsylvania State University, University Park, Pennsylvania 16803, USA \\ ${ }^{2}$ INFN, Sezione Roma 1, Dipartimento di Fisica, Università “La Sapienza," I-00185 Roma, Italy \\ ${ }^{3}$ Université de Lyon, Univ. Lyon 1, CNRS/IN2P3, IPN Lyon, F-69622 Villeurbanne Cedex, France and \\ Theory Group, Physics Department, CERN, CH-1211 Genève 23, Switzerland
}

(Received 2 March 2010; published 21 April 2010)

\begin{abstract}
The kinematics of the decay of a bound proton is governed by the proton spectral function. We evaluate this quantity in ${ }^{16} \mathrm{O}$ using the information from nuclear physics experiments. It also includes a correlated part. The reliability of this evaluation is sufficient to open the possibility of correlated cuts in the missing mass and momentum variables to identify the decay events from the bound protons with a possible increase of the signal-to-noise ratio.
\end{abstract}

DOI: 10.1103/PhysRevC.81.041602

PACS number(s): 13.30.Ce, 24.10.-i, 21.60.-n, 14.20.Dh

Proton decay is an important window for theories beyond the standard model. Several decay channels have been experimentally explored, leading to lower limits for the corresponding proton lifetimes. One of them is the pionic decay mode into a neutral pion and a positron. The signature of a decay process is that the sum of the four momenta of the decay products should reconstitute the proton four-momentum. For a free proton at rest this means a vanishing total three-momentum and a missing mass equal to the free proton mass. The pionic decay experiment at Kamiokande [1,2] is performed with water Cerenkov detectors in such a way that eight in ten decaying protons are embedded in an oxygen nucleus, where their spectrum is modified. In a pure shell model description corrections are applied to incorporate the shellmodel momentum distribution and corresponding binding of the shell model orbits of the oxygen nucleus. However, Yamazaki and Akaishi [3] pointed out that this procedure does not take into account the correlations of the decaying proton with the neighboring nucleons. Using a correlation function deduced from the Reid soft core potential, they evaluated the effect on the invariant mass spectrum which acquires a broad low-energy tail representing $\simeq 10 \%$ of the total decay.

It is customary in the decay problem to introduce the missing three-momentum $P_{\text {miss }}=\Sigma_{i} P_{i}$, the sum of the momenta of the decay particles, and the missing mass, $M_{\text {miss }}^{2}=$ $\left(\Sigma_{i} E_{i}\right)^{2}-\left(\Sigma_{i} P_{i}\right)^{2}$. These quantities also refer to the decaying proton. They are related to the momentum and energy of the residual nucleus, which can be in an excited state defined by $P_{\text {miss }}=P_{A-1}$ and $\Sigma_{i} E_{i}=E_{A-1}^{*}$. Because the state of the residual nucleus for each decay event is not known, there is no strict constraint to identify a proton decay. In the pure shell-model case where the smearing is already present, the effect is rather mild and controllable. However, as shown in Ref. [3], this is not the complete story. It is the aim of this work to evaluate these distributions. The issue at stake is if the broadening is so big that a substantial fraction of genuine decay event is lost in the background. In other words, the lower limit on the proton lifetime deduced from the absence of events within a certain domain in the missing momentum and missing energy variables must take into account the portion of decay events outside this domain. It is therefore useful to have the best possible probability distributions. Our result applies to any decay channel, but for illustrative purposes we will often refer to the pionic channel. It is valid as well for neutron decay with the disappearance of the hadron.

To give a feeling for the importance of the modification introduced by correlations, in terms of particle-hole (ph) excitations, correlations translate into the existence of $2 \mathrm{p} 2 \mathrm{~h}$ excited states mixed into the nuclear ground state. The decay of a correlated particle leaves the nucleus into an excited state with one hole and one particle-one hole, for which we want to evaluate the excitation energy. Beyond the energy associated with one-hole creation as evaluated in the shell model, there is the energy of the particle-hole, which can be approximated by $E_{\mathrm{ph}}=P_{\mathrm{ph}}^{2} / 2 M$, where $P_{\mathrm{ph}}$ is the momentum exchanged between the correlated pair. Neglecting the momentum of the hole which has a relatively narrow distribution, $P_{\mathrm{ph}}$ is also the opposite of the missing momentum.

The missing mass square is then given by

$$
\begin{aligned}
M_{\mathrm{miss}}^{2} & =\left[M^{*}-P_{\mathrm{miss}}^{2} /(2 M)\right]^{2}-P_{\mathrm{miss}}^{2} \\
& \simeq M^{* 2}-2 P_{\mathrm{miss}}^{2},
\end{aligned}
$$

where $M^{*}$ is the nucleon mass reduced by the energy necessary for the hole creation. The missing mass square evolves approximately parabolically with $P_{\text {miss }}$. To illustrate the expected effect, let us take an approximate value $M^{*} \simeq 900 \mathrm{MeV}$. For a typical exchanged momentum $P_{\mathrm{ph}}=300 \mathrm{MeV} / c$, which is also the value of the missing momentum, the missing mass value turns out to be $800 \mathrm{MeV}$. These two missing values happen to be on the borderline of the domain in which Refs. [1,2] interpret an event as a proton decay one (no event, in fact, fell into this domain). We therefore expect that in the analysis a substantial fraction of the correlated decays escapes detection. Moreover, future experiments aiming to improve the current limits on the proton decay will have to introduce even tighter cuts to avoid the background owing to the atmospheric neutrino interactions making the effects discussed even more important. The preceding qualitative argument is made quantitative in the next paragraph.

Because the decay of the bound proton occurs instantaneously on the scale of nuclear interactions, one can express 
the quantities relevant for the bound proton decay in terms of the nuclear spectral function

$$
S_{A}(k, E)=\left\langle\psi_{A}\left|a^{\dagger}(k) \delta\left(H_{A-1}-E\right) a(k)\right| \psi_{A}\right\rangle,
$$

which describes the probability of finding a nucleon in the nucleus with momentum $k$ and producing a residual $A-1$ system with excitation energy $E$ after an instantaneous removal of this nucleon. The spectral function is related to the single nucleon momentum distribution as

$$
\int d E S_{A}(k, E)=n_{A}(k),
$$

and it is normalized as

$$
\int d E d \boldsymbol{k} S_{A}(k, E)=1 .
$$

To resolve the spectral function at the high resolution relevant for the proton decay one needs to use probes which transfer large energies and momenta, above $1 \mathrm{GeV}$, to the nucleons in the nuclei. Such studies were performed in the last few years using proton and electron beams of high energies.

It was observed [4] that the ratios of $\left(e, e^{\prime}\right)$ cross sections off nuclei and the deuteron $\left({ }^{3} \mathrm{He}\right)$ are independent of $x$, $Q^{2}$ for $1.3<x<2$ and $Q^{2} \geqslant 1.5 \mathrm{GeV}^{2}$, corresponding to the kinematics where the electron can scatter only off the correlated nucleon-nucleon pair with internal momenta $\geqslant 300 \mathrm{MeV} / c$. Moreover, in $\left(e, e^{\prime} p\right)$ or $(p, 2 p)$ reactions on nuclei at large $Q^{2}$, a strong correlation was observed between the emission of a fast proton and that of a nucleon (predominantly neutron) in the opposite direction $[5,6]$. These studies confirmed theoretical expectations of the presence of significant short-range correlations (SRCs) in nuclei-for instance, in ${ }^{12} \mathrm{C}$ the probability $P_{{ }^{12}} \mathrm{C}$ of finding a nucleon with momentum $\geqslant 300 \mathrm{MeV} / c$ is a factor of $\sim 5 \pm 0.5$ larger than in the deuteron. The current models of the deuteron give $P_{D}$ in the range $3-4 \%$, and this corresponds to $P_{{ }^{16} \mathrm{O}}=0.15-0.2$. The data also support the expectation that most of this probability is due to the $p n$-tensor correlations (see, e.g., Refs. $[7,8]$ ), which are a specific case of $2 \mathrm{p}-2 \mathrm{~h}$ excitations. For a review and detailed references, see Ref. [9].

In the many-body models of nuclei with realistic $N N$ potential the high-momentum component with momenta between 300 and $600 \mathrm{MeV} / c$ originates from the interplay of attraction and repulsion at distances $\leqslant 1.2 \mathrm{fm}$. Hence, we have used two spectral functions $[10,11]$ calculated in such models to analyze the effect of the nuclear structure on the detection of the bound proton decay.

For the purposes of the analysis of the proton decay events, it is convenient to choose as variables the three-momentum of the decaying proton and the square of the bound proton mass $V=$ $M_{\text {miss }}^{2}$ which fixes its off-shellness. The two spectral functions of Ref. $[10,11]$ have an uncorrelated part, $S_{0}\left(P_{\text {miss }}, E\right)$, and a correlated one, $S_{1}\left(P_{\text {miss }}, E\right)$, where $E$ is the proton removal energy defined as $E=E_{A}-E_{A-1}^{\star}$. In the first model we use $S_{0}$ as calculated with the Skyrme force and renormalized by a factor 0.8 . The correlated part, $S_{1}$ represents $20 \%$ of the total spectral function and it is given by the model of [10]. In this model $\int d^{3} k d E S_{1}(k, E) \theta\left(k-k_{0}\right)=11 \%$, where $k_{0}=$
$300 \mathrm{MeV} / c$ (the second model we considered provides the same result as far as this quantity is concerned). The ratio of ${ }^{16} \mathrm{O}$ and deuteron high-momentum components in these models varies in the range of 3-6 for $300<k<600 \mathrm{MeV} / c$, which is rather close to the value of the ratio $\sim 5$ obtained from the analysis of the hard phenomena and in particular $x>1$ data (see the review in Ref. [9]). The smaller value of the total probability than in a phenomenological estimate is mainly attributable to a later onset of the dominance of the SRC regime. The model of Ref. [10] for the correlated part of the spectral function is based on the notion of the factorization of the two-body momentum distribution for high values of the relative and small values of the center-of-mass momenta of the pair and it is valid in this region; this factorization was justified within a many-body approach in Ref. [12] and shown to hold for ${ }^{16} \mathrm{O}$ within the many-body calculation of Ref. [7]. It also gives a correct dependence for the center of mass of the correlated pair, as measured in Refs. [5] and [6]. We choose for the relative motion of the pair in the two-body momentum distribution a parametrization which reproduces well the high-momentum tail of the deuteron in the region of interest and leads to a good description of the high-momentum tail of $n_{{ }^{16} \mathrm{O}}(k)[13,14]$.

The spectral function of Ref. [11] has been obtained within the local density approximation [15], in which the $\left(e, e^{\prime} p\right)$ data on single nucleon knock-out at low missing energy [16] is combined with the results of accurate theoretical calculations of the nuclear matter spectral function at different densities [17]. A direct measurement of the correlation component of the spectral function of ${ }^{12} \mathrm{C}$, obtained measuring the $\left(e, e^{\prime} p\right)$ cross section at missing momentum and energy up to $\sim 800$ and $\sim 200 \mathrm{MeV}$, respectively, has been carried out recently at Jefferson Lab by the E97-006 Collaboration [18]. The data resulting from the preliminary analysis appear to be consistent with the theoretical predictions based on the spectral function of Ref. [11].

The quantity $P_{\text {miss }}^{2} S_{0}\left(P_{\text {miss }}, V\right)$ is shown in Figs. 1 and 2 in three-dimensional plots, and $P_{\text {miss }}^{2} S_{0}\left(P_{\text {miss }}, M_{\text {miss }}\right)$ is shown in Figs. 3 and 4 in contour plots for the two considered models. The strength is concentrated over three or four stripes in the $P_{\text {miss }}, M_{\text {miss }}$ plane in the two cases; they correspond to the occupied shells of ${ }^{16} \mathrm{O}$ : the $P_{1 / 2}, P_{3 / 2}$, and $S_{1 / 2}$ states in the case of calculation with the Skyrme force and to an additional $P_{3 / 2}$ state in the case of Ref. [11].

The energies and widths of the occupied states in the two models we considered are as follows. In the first model, as calculated from the Hartree-Fock Skyrme model with shell-model parameters which describe the $(p, 2 p)$ and $(p, p n)$ data of Refs. $[19,20]$, they are $12.06 \mathrm{MeV}$ with a width $\simeq 5 \mathrm{MeV}$ for the $P_{1 / 2}$ state and the $P_{3 / 2}$ state has 18.63 $\mathrm{MeV}$ with a width of $5 \mathrm{MeV}$; the $S$ state is quite broad with a width of $\simeq 40 \mathrm{MeV}$ for an energy $37.96 \mathrm{MeV}$. In the second model (Ref. [11]), we have the following energies, with roughly the same widths: $P_{1 / 2}$ at $12.50 \mathrm{MeV}, P_{3 / 2}$ at $18.75 \mathrm{MeV}$, a second $P_{3 / 2}$ at $23.00 \mathrm{MeV}$, and $S_{1 / 2}$ at 42.50 $\mathrm{MeV}$. The model takes into account a rather small contribution of higher-energy excitations where intermediate states with one particle in the continuum and one hole in the final state. 

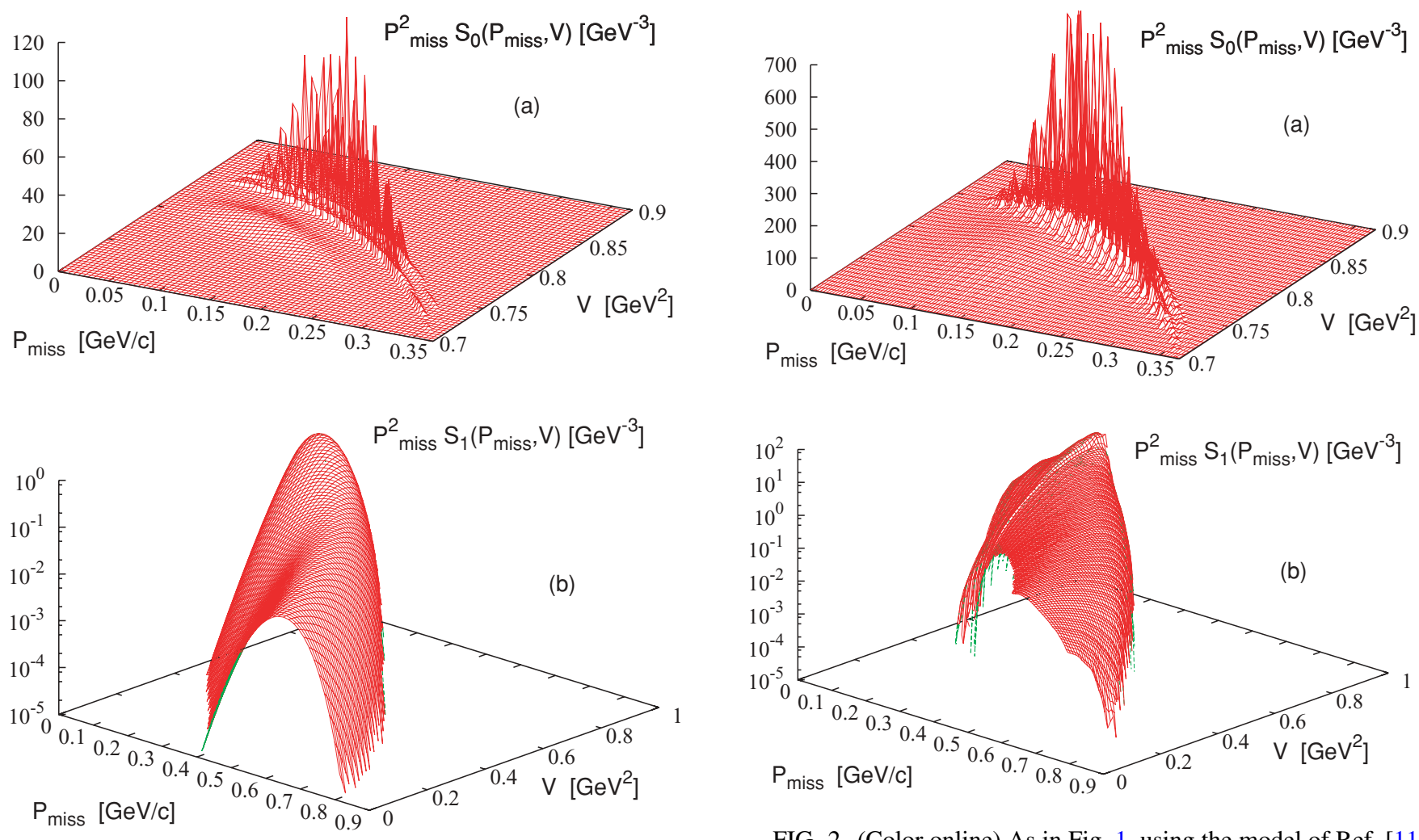

FIG. 1. (Color online) (a) The uncorrelated proton spectral function of ${ }^{16} \mathrm{O}, P_{\text {miss }}^{2} S_{0}\left(P_{\text {miss }}, V\right)$, as calculated with the Skyrme force and plotted vs missing momentum $P_{\text {miss }}$ and virtuality, $V=M_{\text {miss }}^{2}$. (b) The correlated proton spectral function of ${ }^{16} \mathrm{O}$ from Ref. [10], $P_{\text {miss }}^{2} S_{1}\left(P_{\text {miss }}, V\right)$.

The behavior in the momentum $P_{\text {miss }}$ can be inferred from the expression of $V$, which in the uncorrelated case is

$$
\begin{aligned}
V & \equiv M_{\mathrm{miss}}^{2}=\left(P_{\mu A}-P_{\mu A-1}\right)^{2} \\
& =\left(M_{p}-E-\frac{P_{\mathrm{miss}}^{2}}{2 M_{A-1}}\right)^{2}-P_{\mathrm{miss}}^{2} .
\end{aligned}
$$

Expanding the square in Eq. (5) and neglecting the $P_{\text {miss }}^{4}$ term, we obtain

$$
\begin{aligned}
V\left(P_{\mathrm{miss}}\right) & \simeq\left(M_{p}-E\right)^{2}-\frac{M_{p}-E+M_{A-1}}{M_{A-1}} P_{\mathrm{miss}}^{2} \\
& =\left(M_{p}-\epsilon_{\alpha}\right)^{2}-C_{\alpha} P_{\mathrm{miss}}^{2}
\end{aligned}
$$

where $\epsilon_{\alpha}$ are the values of the proton shells energies. The coefficients $C_{\alpha}$ are 1.07 and 1.06 for the $P$ and $S$ proton shells, respectively. As for the correlated spectral function, $P_{\text {miss }}^{2} S_{1}\left(p_{\text {miss }}, V\right)$ is represented in Figs. 1 and 2 in threedimensional plots, and $P_{\text {miss }}^{2} S_{1}\left(p_{\text {miss }}, M_{\text {miss }}\right)$ is shown in Figs. 3 and 4 in contour plots; it has a similar behavior to the corresponding uncorrelated quantity, but it is broader. One can see from Figs. 3 and 4 that the correlated spectral functions in the two models exhibit differences; a detailed comparison of the two spectral functions is out of the scope of the present article. Nevertheless, these differences do not affect our conclusions, which are given in the following, because most

FIG. 2. (Color online) As in Fig. 1, using the model of Ref. [11] for both the uncorrelated and the correlated parts.

of the strength is concentrated along the stripe of maximum strength, even if the second model is more peaked at low momenta and it is more narrow around the center of the stripe. The center of the corresponding stripe obeys the following equation in the $V, P_{\text {miss }}$ plane for both models:

$$
V\left(P_{\text {miss }}\right)=0.78-1.78 P_{\text {miss }}^{2},
$$

where $V$ is expressed in $\mathrm{GeV}^{2}$ and $P_{\text {miss }}$ in $\mathrm{GeV} / c$, which is close to the approximate expression of Eq. (1).

The concentration of the strength of the spectral function in limited regions of space which project in some bands in the $M_{\text {miss }}, P_{\text {miss }}$ plane suggests a complementary analysis of the data specifically aimed at the decay of the ${ }^{16} \mathrm{O}$ protons. It consists of the following: to look for events which, in this plane, fall in one or several, depending of the accuracy of the data, regions of this plane selected to cover the lines of the maximum of the (uncorrelated or correlated) strengths defined in Eqs. (6) and (7) so as to maximize the number of significant events while minimizing the background, that is, the area. Correlated events can also be included in this way. The calculated proton spectral function in ${ }^{16} \mathrm{O}$ is sufficiently reliable, because it is established in connection with various nuclear physics experiments, to allow for this possibility.

This kind of analysis, if feasible, precludes the subsequent distortions of the pion kinematics after emission by the proton. We comment later on that. A technical remark: For the correlated part, when transforming coordinates from $\left(P_{\text {miss }}, E\right)$ to $\left(P_{\text {miss }}, V\right)$ as in Eq. (5), we impose that $V$ stays positive, so we forbid a certain region of $E, P_{\text {miss }}$ space to be accessible; this in turn means the normalization of the correlated spectral 

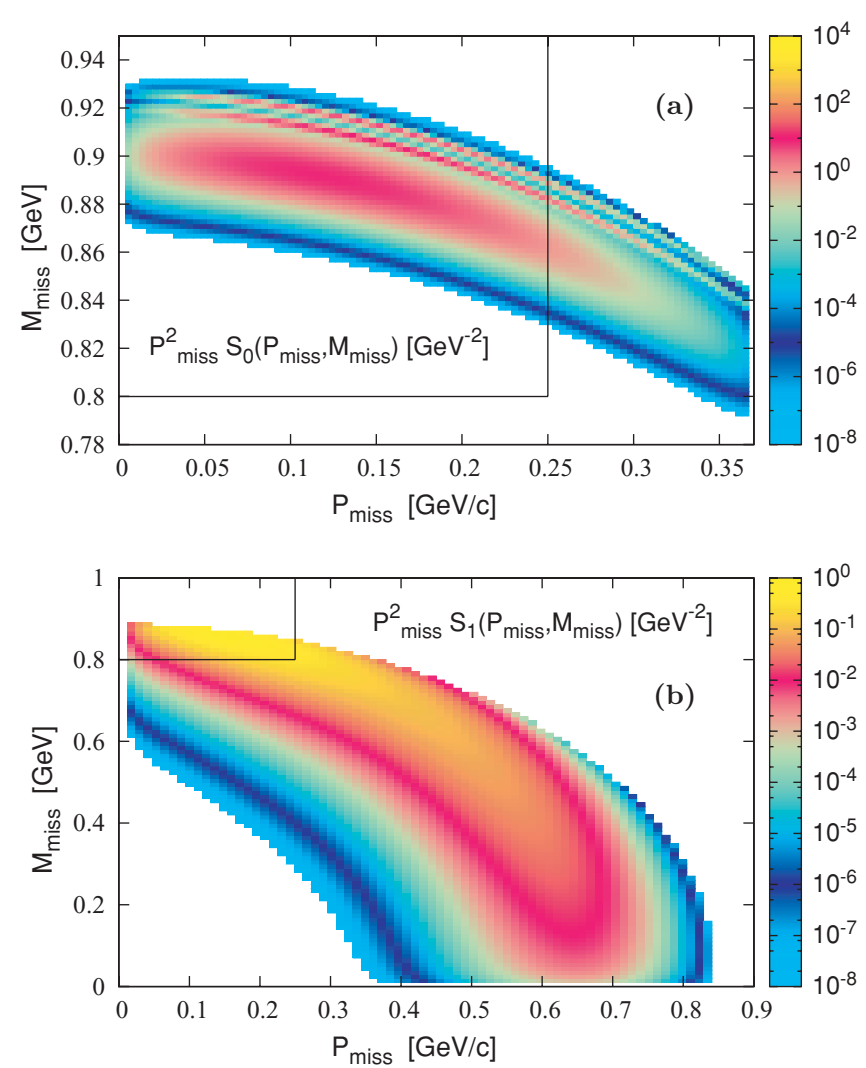

FIG. 3. (Color online) (a) Contour plot of the uncorrelated proton spectral function of ${ }^{16} \mathrm{O}, P_{\text {miss }}^{2} S_{0}\left(P_{\text {miss }}, M_{\text {miss }}\right)$, as calculated with the Skyrme force and plotted vs missing momentum $P_{\text {miss }}$ and missing mass $M_{\text {miss }}=\sqrt{V}$. (b) The correlated proton spectral function of ${ }^{16} \mathrm{O}$ from Ref. [10], $P_{\text {miss }}^{2} S_{1}\left(P_{\text {miss }}, M_{\text {miss }}\right)$. We show with black solid lines the cut $P_{\text {miss }}<250 \mathrm{MeV}$ and $V<640 \mathrm{MeV}$, quoted in Refs. [1] and [2], in both panels.

function is not exactly $0.2=\int d E d \boldsymbol{P}_{\text {miss }} S_{1}\left(P_{\text {miss }}, E\right)$, but

$$
\int d V d \boldsymbol{P}_{\text {miss }} S_{1}\left(P_{\text {miss }}, V\right)=0.18 \text {. }
$$

We have checked that integrating over negative values of $V$ gives the missing normalization, $\int_{-\infty}^{0} d V \int d \boldsymbol{P}_{\text {miss }} S_{1}$ $\left(P_{\text {miss }}, V\right)=0.02$.

In Fig. 5 we show the total spectral function $S_{0}+S_{1}$, in the two considered models, as a function of $P_{\text {miss }}$ and $M_{\text {miss }}$, while in Fig. 6 we present the normalization integral of the spectral function

$$
N\left(V_{\max }\right)=\int_{0}^{V_{\max }} d V \int d \boldsymbol{P}_{\text {miss }} S\left(P_{\text {miss }}, V\right) .
$$

It represents the number of events lost by applying a cut on the missing mass such that only the events that correspond to a missing mass larger than this particular value $M_{\text {miss }}$ are kept, irrespective of the momentum. If no cut on $V$ is applied, $N=0.98$. If we use the cut $\sqrt{V} \geqslant 800 \mathrm{MeV}, k \leqslant$ $250 \mathrm{MeV} / c$, presented in Figs. 3, 4, and 5, the number of nuclear events is reduced by a factor of $N=0.83$ using the model of Ref. [10] and by a factor of $N=0.80$ using the model of Ref. [11]. However, future experiments are likely to have to introduce tighter cuts to reduce the background
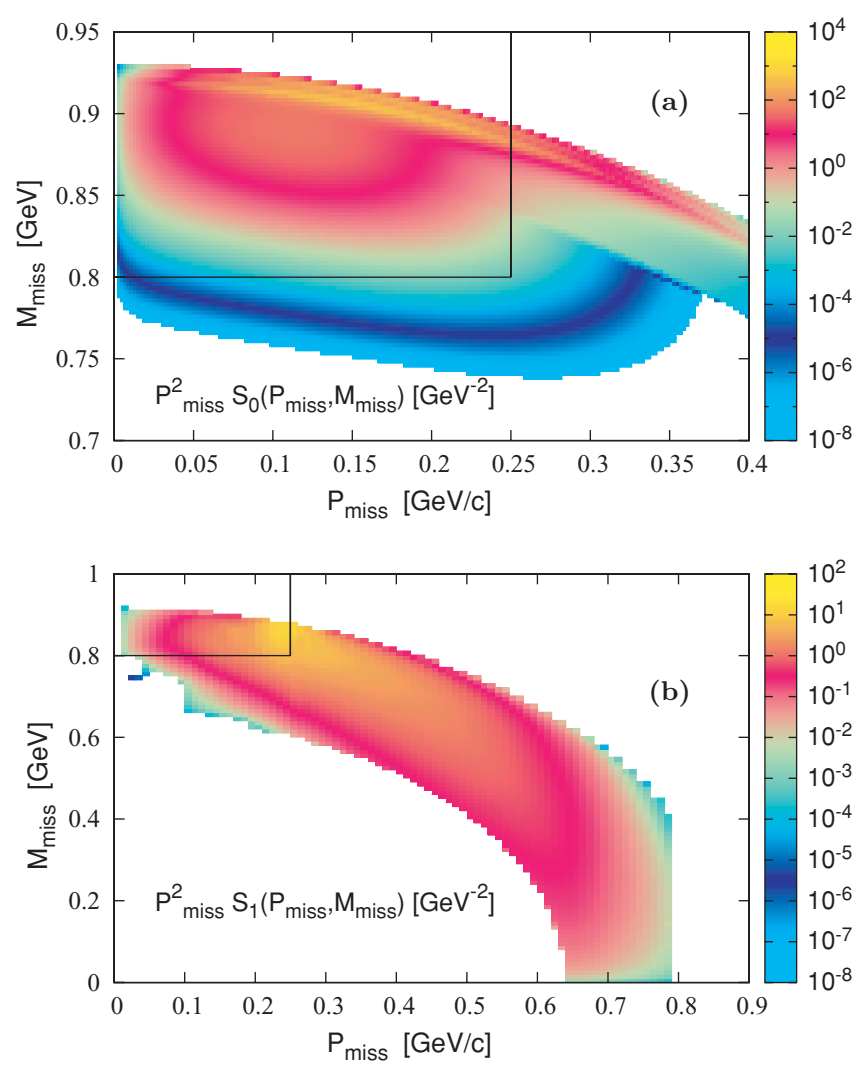

FIG. 4. (Color online) As in Fig. 3, but with the model spectral function of Ref. [11].

from the interactions of atmospheric neutrinos. If, for example, the cut $\sqrt{V}>900 \mathrm{MeV}$ is imposed, $\simeq 44 \%$ of the events are removed, namely, $26 \%$ (25\%) from uncorrelated events and $18 \%(19 \%)$, the near totality of correlated events in the first (second) considered model. With a tight constraint on $M_{\text {miss }}$, the fraction lost is quite appreciable if no other precaution is taken. The correlated analysis that we discussed may allow a better efficiency.

There are other effects that reduce the contribution of the bound nucleon decays. This includes a reduced-phase volume that is $\propto \sqrt{V}$ for decays with the production of light particles. Furthermore, the very mechanism of the proton decay may be sensitive to the nuclear correlations. For example, if the decay amplitude is proportional to the three-quark wave function at the origin (see, e.g., Ref. [21]), the effect of suppression of the pointlike configurations in bound nucleons [22,23] would contribute, reducing the rate of the decay by about $14 \%$ for the $M_{p}-\sqrt{V}=100 \mathrm{MeV}$ cut.

All the effects that we have discussed are genuine medium effects on the decay amplitude. They are not the whole story. The subsequent history of the pion, rescatterings and absorption in oxygen, further reduces the number of "observable" pions. The inelastic scattering of pions clouds the message on the kinematics because the inelastically scattered pion ejects a nucleon. The corresponding point in the $M_{\text {miss }}, P_{\text {miss }}$ plane would be likely to fall outside the interesting regions delimited from the proton spectral function that we discussed in this work. Therefore, for an analysis of the type suggested in this work, inelastically scattered pions may be considered as lost 

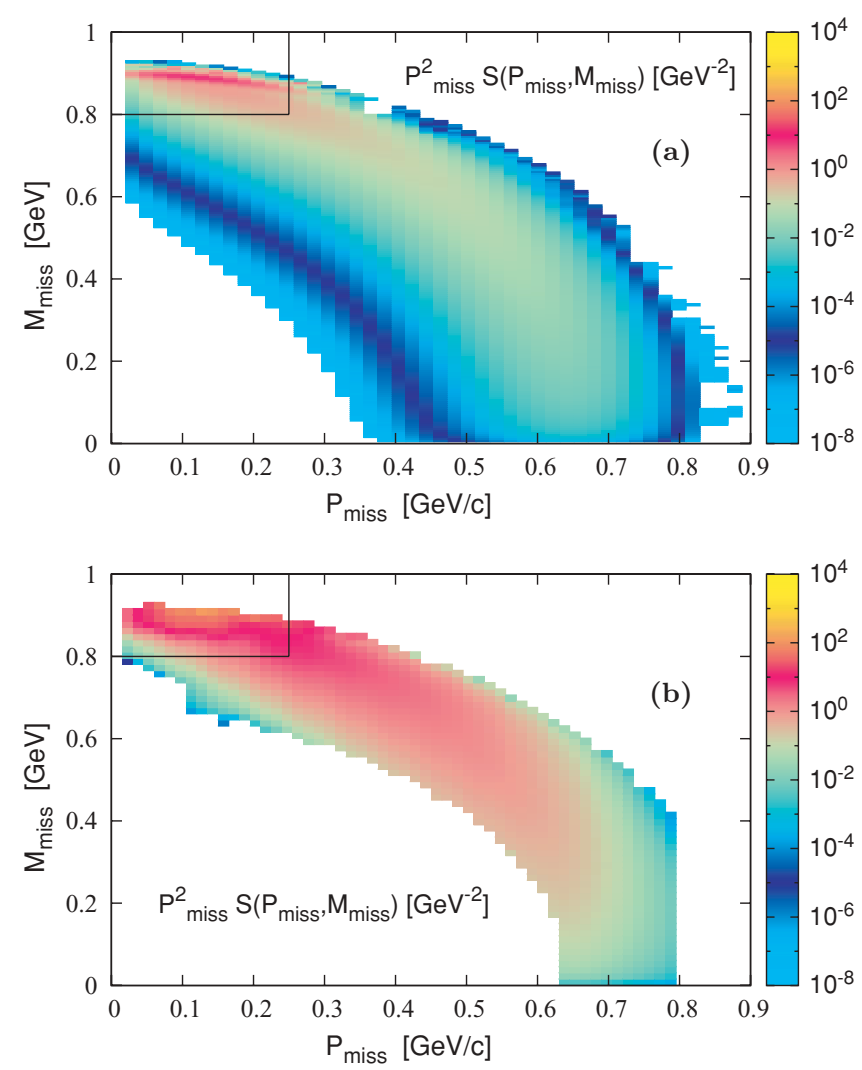

FIG. 5. (Color online) The total spectral function, $S_{0}+S_{1}$, within the two considered models. Panel (a) corresponds to the mixed model of $S_{0}$ from the Skyrme force and $S_{1}$ from Ref. [10]. Panel (b) is the model of Ref. [11].

events. It may represent a reduction factor of about 0.6 . This is usually taken care of through a Monte Carlo evaluation, which is beyond the scope of the present work.

In conclusion, we have introduced, in the problem of the identification of the decay events of the protons bound in the oxygen nucleus, the use of proton spectral function. It allows the prediction of the location in the $M_{\text {miss }}, P_{\text {miss }}$ plane of the decay events. Our spectral function has an uncorrelated part and a correlated part. It is has been tested against a number of nuclear physics experiments and the reliability of our prediction is sufficient to be exploitable. We considered two models for the spectral function and the conclusions on

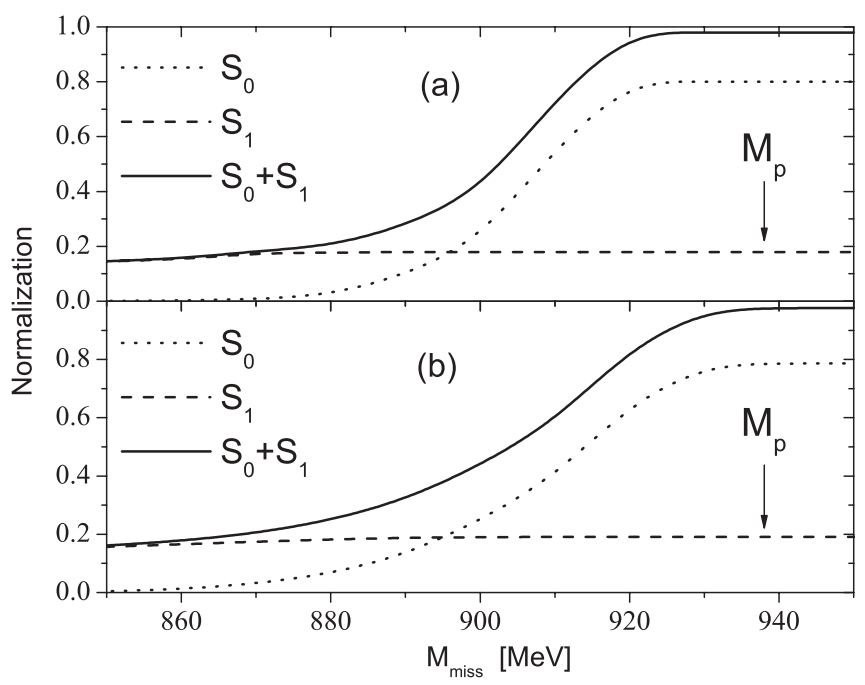

FIG. 6. The normalization of the spectral function after the cut of Eq. (1), as a function of the upper limit of integration in $\left(M_{\operatorname{miss}}\right)_{\max }=$ $\sqrt{V_{\max }}$; the arrow shows the position of the proton mass, $M_{p}$. Panel (a) corresponds to the mixed model of $S_{0}$ from the Skyrme force and $S_{1}$ from Ref. [10], and panel (b) corresponds to the model of Ref. [11]; separate contributions are shown for the uncorrelated and correlated parts within both models.

the correlated cuts holds even if the two models exhibit some difference for the spectral functions. It appears that for the future nucleon decay experiments with tight cuts on the mass of the products of the proton decay, it may be interesting to consider, as complementary information for the decay events of the oxygen protons, correlated cuts on the mass and missing momentum obtained from the spectral function to decrease the background-to-signal ratio. The price to pay for this type of analysis is the loss of decay events where a pion produced in the decay is inelastically scattered, which clouds the reconstitution of the proton spectral function in oxygen. The loss in intensity, however, is moderate and it may be compensated by the advantage of a decrease of the background.

We thank G. Chanfray, C. Ciofi degli Atti, D. Davesne, M. Fidecaro, L. Frankfurt, M. Martini, and M. Zhalov for useful discussions. The work of M.A. and M.S. is supported by DOE under Contract DE-FG02-93ER40771.
[1] M. Shiozawa et al. (Super-Kamiokande Collaboration), Phys. Rev. Lett. 81, 3319 (1998).

[2] H. Nishino et al. (Super-Kamiokande Collaboration), Phys. Rev. Lett. 102, 141801 (2009).

[3] T. Yamazaki and Y. Akaishi, Phys. Lett. B 453, 1 (2000).

[4] K. S. Egiyan et al. (CLAS Collaboration), Phys. Rev. Lett. 96, 082501 (2006).

[5] R. Shneor et al. (Jefferson Lab Hall A Collaboration), Phys. Rev. Lett. 99, 072501 (2007).

[6] A. Tang et al., Phys. Rev. Lett. 90, 042301 (2003).

[7] M. Alvioli, C. Ciofi degli Atti, and H. Morita, Phys. Rev. Lett. 100, 162503 (2008).
[8] R. Schiavilla, R. B. Wiringa, S. C. Pieper, and J. Carlson, Phys. Rev. Lett. 98, 132501 (2007).

[9] L. Frankfurt, M. Sargsian, and M. Strikman, Int. J. Mod. Phys. A 23, 2991 (2008).

[10] C. Ciofi degli Atti and S. Simula, Phys. Rev. C 53, 1689 (1996).

[11] O. Benhar, N. Farina, H. Nakamura, M. Sakuda, and R. Seki, Phys. Rev. D 72, 053005 (2005).

[12] M. Baldo, M. Borromeo, and C. Ciofi degli Atti, Nucl. Phys. A 604, 429 (1996).

[13] M. Alvioli, C. Ciofi degli Atti, and H. Morita, Phys. Rev. C 72, 054310 (2005). 
[14] S. C. Pieper, R. B. Wiringa, and V. R. Pandharipande, Phys. Rev. C 46, 1741 (1992).

[15] O. Benhar, A. Fabrocini, S. Fantoni, and I. Sick, Nucl. Phys. A 579, 493 (1994).

[16] S. Turck-Chièze, Lect. Notes Phys. 137, 251 (1981).

[17] O. Benhar, A. Fabrocini, and S. Fantoni, Nucl. Phys. A 505, 267 (1989).

[18] D. Rohe et al., Phys. Rev. Lett. 93, 182501 (2004).

[19] S. L. Belostotsky et al., in Proceedings of the International Symposium on Modern Developments in Nuclear Physics,
June 27-July 1, 1987, Novosibirsk 1987, edited by O. P. Sushkov (World Scientific, Singapore, 1988), p. 191.

[20] N. Liyanage et al. (Jefferson Lab Hall A Collaboration), Phys. Rev. Lett. 86, 5670 (2001).

[21] V. S. Berezinsky, B. L. Ioffe, and Y. I. Kogan, Phys. Lett. B 105, 33 (1981).

[22] L. L. Frankfurt and M. I. Strikman, Nucl. Phys. B 250, 143 (1985)

[23] M. R. Frank, B. K. Jennings, and G. A. Miller, Phys. Rev. C 54, 920 (1996). 